\title{
Highly Sensitive ZnO NWFET Biosensor Fabricated Using Top-Down Processes
}

\author{
N.M.J. Ditshego \\ Electrical, Computer and Telecommunications Engineering Department, FET, \\ Botswana International University of Science and Technology (BIUST), \\ Private bag 16, Palapye, Botswana \\ Email: ditshegon@biust.ac.bw
}

Keywords: zinc oxide, field effect transistor, nanowire, device, nanosensor, lysozyme (LYSO), phosphate buffered saline (PBS), bovine serum albumin (BSA)

\begin{abstract}
A highly sensitive low-doped ZnO nanowire field effect transistor (NWFET) biosensor has been fabricated and measured. The low doped biosensor with NWFET transducer was used to sense charge of the following substances: lysozyme (LYSO), phosphate buffered saline (PBS), bovine serum albumin (BSA). It achieved maximum sensitivity of $-543.2 \%$ for the PBS-LYSO protein and 13,069 \% for the PBS-BSA protein. These results were achieved because the electrical measurement and characterisation was focused on the charge effect of the LYSO and BSA acting on the $\mathrm{ZnO}$ nanowire subthreshold region. The nano-fabrication process is stable and reproducible. The high sensitivity of the ZnO NWFET biosensor can be exploited for selective analyte detection by functionalizing the nanowire surface with antibodies and/or other biomolecular probe molecules.
\end{abstract}

\section{Introduction}

Researchers [1-10] continue to investigate zinc oxide nanowire field effect transistor (NWFET) transducer as a sensing agent for protein molecules. The task of the transducer is to convert the charge of protein molecules into electrical signal that can then be transmitted for processing [11, 12, 13]. The device has practical advantages: low costs, abundant, non-toxic, transparent, large excitonic binding energy of $60 \mathrm{meV}$, soluble, compatible with intercellular material, and wide and direct band gap of $3.37 \mathrm{eV}$ making it highly sensitive [14-19]. These advantages make it a highly attractive sensor for the future semiconductor industry.

The wide band gap is highly desirable as it increases sensitivity. To further increase device sensitive, three things are investigated: gate oxide made of $\mathrm{Al}_{2} \mathrm{O}_{3}$, low doping and the nanowire structure $[1,20]$ of the $\mathrm{ZnO}$ channel. $\mathrm{Al}_{2} \mathrm{O}_{3}$ will be used because it is compatible with $\mathrm{ZnO}$ and has a high- $k$ dielectric constant. The high- $k$ dielectric constant allows for the oxide layer thickness to be increased without sacrificing sensitivity. Low doping of the semiconductor material increases sensitivity because it is inversely proportional to device sensitivity. This is described in equation 1 [1, 20-22] below:

$$
\text { Sensitivity }(S)=\frac{\Delta G}{G_{0}}=\frac{2 \varepsilon_{r} \varepsilon_{0} \emptyset_{B} N(t)}{q a^{2} N_{D} \log \left(1+\frac{t_{D}}{a}\right)}
$$

The parameters of the equation are defined as follows: $\Delta \mathrm{G}$ is the change in conductivity of the device, $\mathrm{G}_{0}$ is the initial conductivity, $\emptyset_{B}$ is the surface potential, $\mathrm{N}(\mathrm{t})$ is the density of charge states at the surface, 'a' is a geometry parameter, $\varepsilon_{r}$ is the effective dielectric constant of $\mathrm{Al}_{2} \mathrm{O}_{3}$, and $\mathrm{N}_{\mathrm{D}}$ is the donor concentration. Thirdly, nanowires improve sensitivity because they are comparable to most biological entities and possess very large surface to volume ratios [20-25]. Due to top-down fabrication processes engineers will have control over location, orientation and dimensions of device on wafer [3, 4, 26-28]. 
This paper presents a highly sensitive biosensor that uses top-down passivated ZnO NWFET as a transducer. The high sensitivity is achieved because the electrical measurements and characterisation focuses on the charge effect of the lysozyme from chicken white (LYSO: Fluka 62970) and bovine serum albumin (BSA: Sigma A3059) acting on the ZnO nanowire subthreshold region which possess a logarithmic scale. The subthreshold voltage shift due to the proteins is used: to extract the charge sensitivity, determine protein charge polarity and estimate the charge distribution.

\section{Experiment}

Fig. 1 shows a schematic diagram of the biosensor configuration. The experimental procedure for the $\mathrm{ZnO}$ NWFET and its application as a biosensor is as described in [5]. The recipe [5] was altered so as to improve sensitivity. The main improvement made was to the remote plasma atomic layer deposition (RPALD) process. All other steps were kept relatively the same. RPALD is capable of producing highly conformal and quality films [3]. The process is cyclic and is based on the number of reactants. For $\mathrm{ZnO}$ films, the cycle depends on two reactants: metallisation and oxidation. Metallisation uses diethyl zinc (DEZ) as the zinc ( $\mathrm{Zn})$ metal precursor. Purge and pump steps are used to separate the execution of the reactants and to remove any by-products. Before deposition: the wafer (substrate) is pre-heated to a temperature used for deposition $\left(190{ }^{\circ} \mathrm{C}\right)$ and it is also cleaned with an $\mathrm{O}_{2}$ plasma so as to remove any polymer layer. During the metallisation step, the DEZ $\left(\mathrm{Zn}\left(\mathrm{C}_{2} \mathrm{H}_{5}\right)_{2}\right)$ is absorbed onto the surface of the wafer, then on another step, $\mathrm{O}_{2}$ is delivered to react with the absorbed DEZ [3].

Four main parameters were altered: film thickness, DEZ dose time, $\mathrm{O}_{2}$ dose time and the order of gas flow. DEZ dose time was reduced from a value of $1.0 \mathrm{~s}$ to a value of $50 \mathrm{~ms}$, while $\mathrm{O}_{2}$ dose time was reduced from $4.0 \mathrm{~s}$ to $2.65 \mathrm{~s}$. The doping concentration [3] in $\mathrm{ZnO}$ depends on oxygen defects (oxygen interstitials $\left(\mathrm{O}_{\mathrm{i}}\right)$, oxygen antisite defects $\left(\mathrm{O}_{\mathrm{Zn}}\right)$, and oxygen vacancies $\left(\mathrm{V}_{\mathrm{o}}\right)$ ). The recipe [5] had more $\mathrm{Zn}$ than $\mathrm{O}$. By reducing the DEZ dose time from $1.0 \mathrm{~s}$ to $50 \mathrm{~ms}$, it caused the $\mathrm{O}$ content to be much higher than the $\mathrm{Zn}$ content which increased the doping concentration. This means oxygen interstitials $\mathrm{O}_{\mathrm{i}}$ and oxygen antisite defects $\mathrm{O}_{\mathrm{Zn}}$ have more effect on the doping concentration than oxygen vacancies $\mathrm{V}_{\mathrm{o}}$. To reduce the interface state charge, the $\mathrm{ZnO}$ deposition recipe was modified. Instead of flowing $\mathrm{O}_{2}$ first into the chamber, DEZ was flowed first. An $\mathrm{O}_{2}$ plasma tends to damage the $\mathrm{SiO}_{2}$ surface, thereby increasing the amount of interface state charge. The deposition of a zinc layer first provides some protection against surface damage from the $\mathrm{O}_{2}$ plasma.

The device was used to measure three aqueous solutions: bovine serum albumin (BSA: Sigma A3059 at concentration of $0.5 \mu \mathrm{M}$ ), lysozyme from chicken white (LYSO: Fluka 62970 at concentration of $0.5 \mu \mathrm{M}$ ) and phosphate buffered saline (pure-PBS) $(150 \mu \mathrm{M} \mathrm{NaCl}, 10 \mu \mathrm{M}$ phosphate, $\mathrm{pH} 7.4$ ), [5]. The electrical I-V characterization of the ZnO NWFETs was done using an Agilent Technologies B1500A semiconductor parametric analyser [5]. All protein detection measurements were carried out using a grounded reference electrode. Four probes were used to measure the characteristics. The probes were for the source, drain, back-gate and top-gate. The procedure for protein detection in this paper is comparable to other authors $[1,11,13,22,24,25]$. 


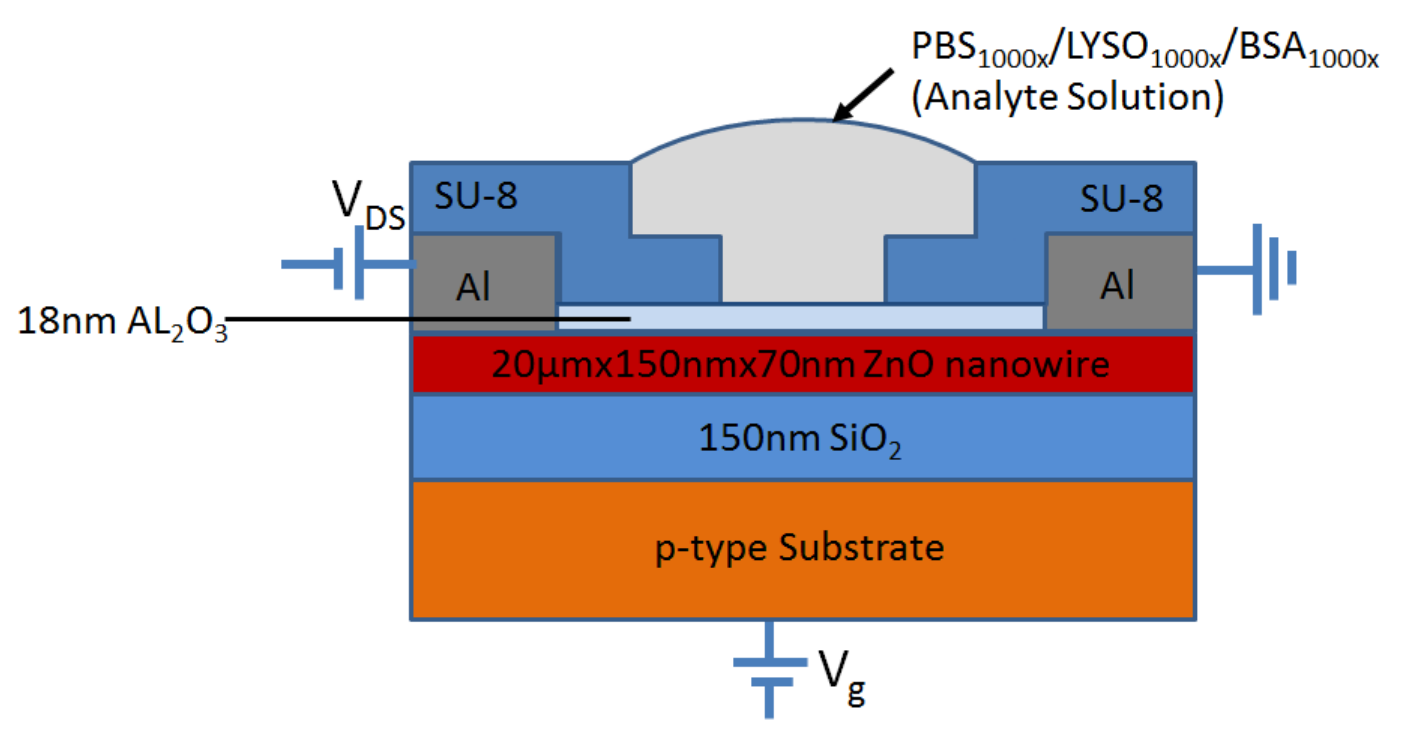

Fig. 1: Schematic illustration of the fabricated biosensor.

Fig. 2 shows SEM cross-sectional image of the $\mathrm{ZnO}$ nanowire device. The low doped device was found to have a channel length of $20 \mu \mathrm{m}$, a height of $150 \mathrm{~nm}$ and a width of $70 \mathrm{~nm}$. The width of the nanowire was measured halfway down the nanowire which is the average of the widths measured at different heights.

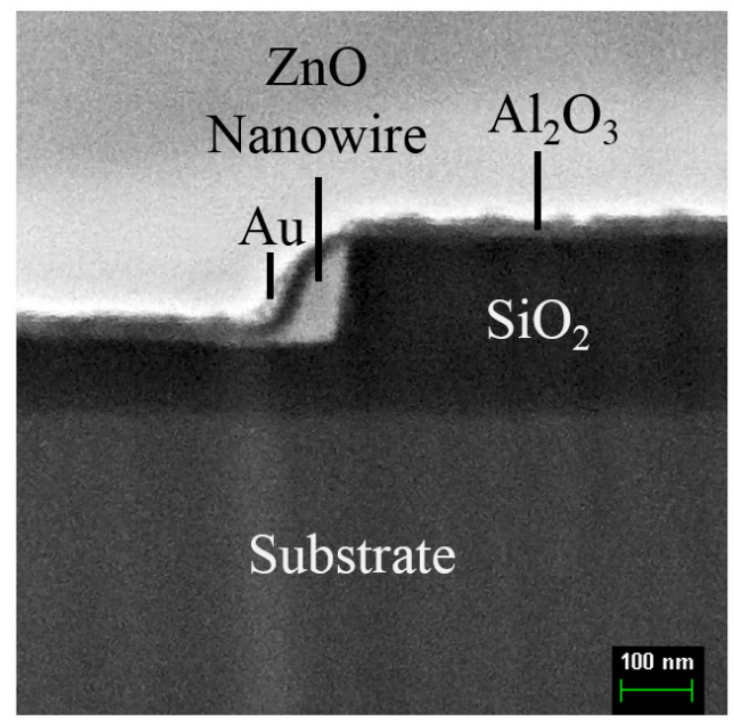

Fig. 2: Cross-sectional SEM images of $\mathrm{ZnO}$ nanowire transducer.

\section{Procedure for protein sensing}

Four different environments were measured: AIR, PBS, lysozyme from chicken white (LYSO: Fluka 62970), bovine serum albumin (BSA: Sigma A3059) [5]. Measurements were carried out in air, then in liquid and then in air again. To measure in liquid, a $5 \mu 1$ droplet of the aqueous solution was introduced in the SU-8 well so as to make contact with channel. There is a wait period between measurements where the analyte solution is allowed to soak onto the surface. The low doped biosensor was measured at short time intervals of five minutes. Each environment is measured five times, once every five minutes. Table 1 shows the sequence of measuring the biosensor. The mechanism of the protein adsorption on the $\mathrm{Al}_{2} \mathrm{O}_{3}$ surface is in equilibrium with the protein concentration in solution. 
Table 1: Experimental procedure that describes the sequence of measuring the low doped biosensor for four different environments (AIR, PBS, lysozyme from chicken white (LYSO: Fluka 62970), bovine serum albumin (BSA: Sigma A3059)) [5].

\begin{tabular}{|c|c|}
\hline Bio-Sensor & Procedure for protein sensing \\
\hline $\begin{array}{l}\text { Low doped } \\
\text { device }\end{array}$ & 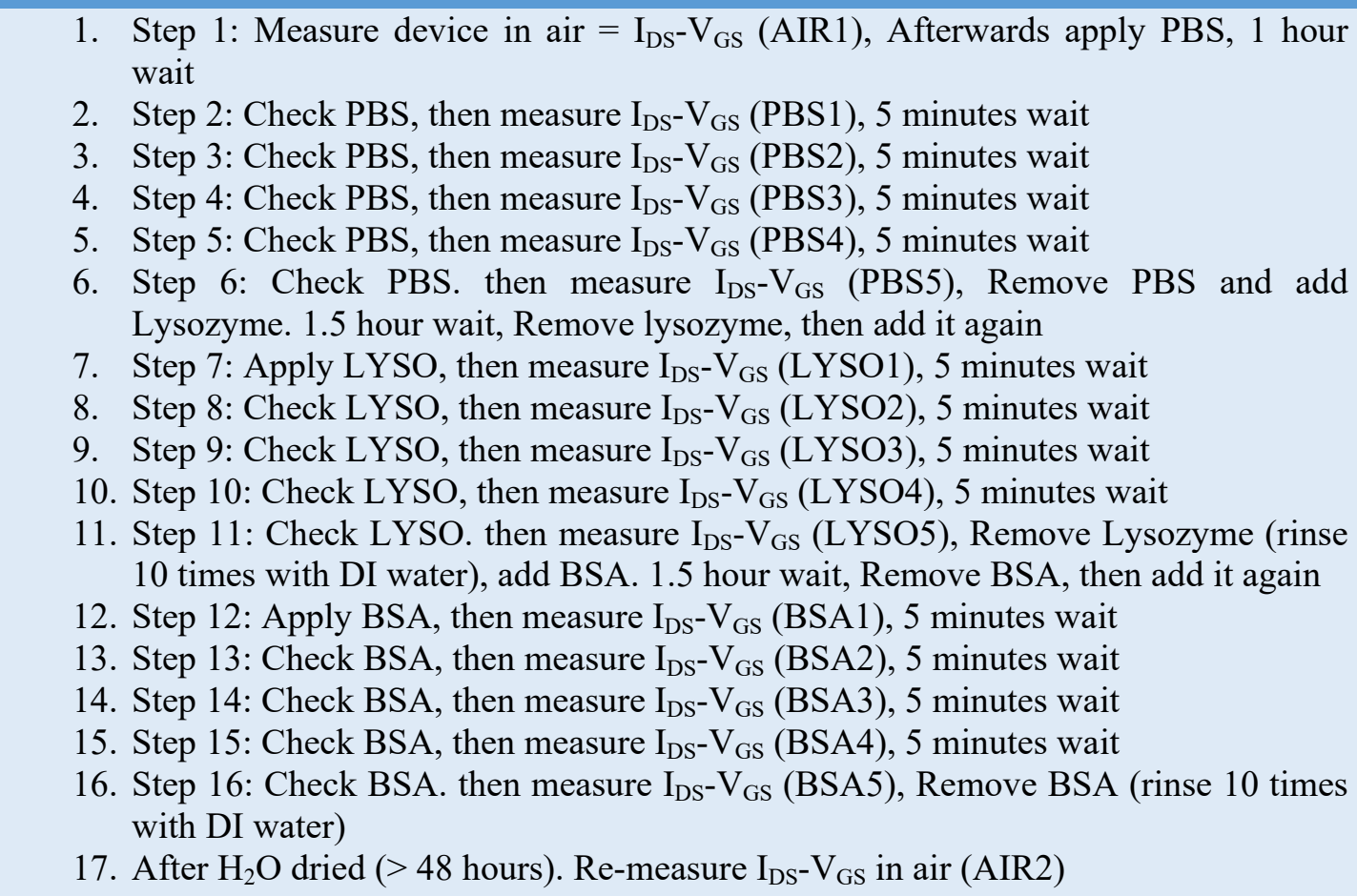 \\
\hline
\end{tabular}

\section{Results}

Fig. 3 shows the electrical characteristics of the low doped device in air before analyte solution measurements. Fig. 3 (a) shows $\mathrm{I}_{\mathrm{DS}} \mathrm{V}_{\mathrm{DS}}$ biosensor measurements in air and darkness with gate bias varying from $-20 \mathrm{~V}$ to $0 \mathrm{~V}$ in steps of $2.0 \mathrm{~V}$ and drain bias from $0 \mathrm{~V}$ to $40 \mathrm{~V}$. It shows clear linear, pinch-off, and saturation regions. Fig. 3 (b) is the subthreshold and linear $\mathrm{I}_{\mathrm{DS}} \mathrm{V}_{\mathrm{GS}}$ plot of the NWFET in air before being exposed to the analytes. The characteristics were measured with $V_{D S}$ at $12.0 \mathrm{~V}$. The device has a subthreshold slope of $170 \mathrm{mV} /$ decade and a threshold voltage of $-2.5 \mathrm{~V}$, obtained by extrapolation of the linear characteristic.

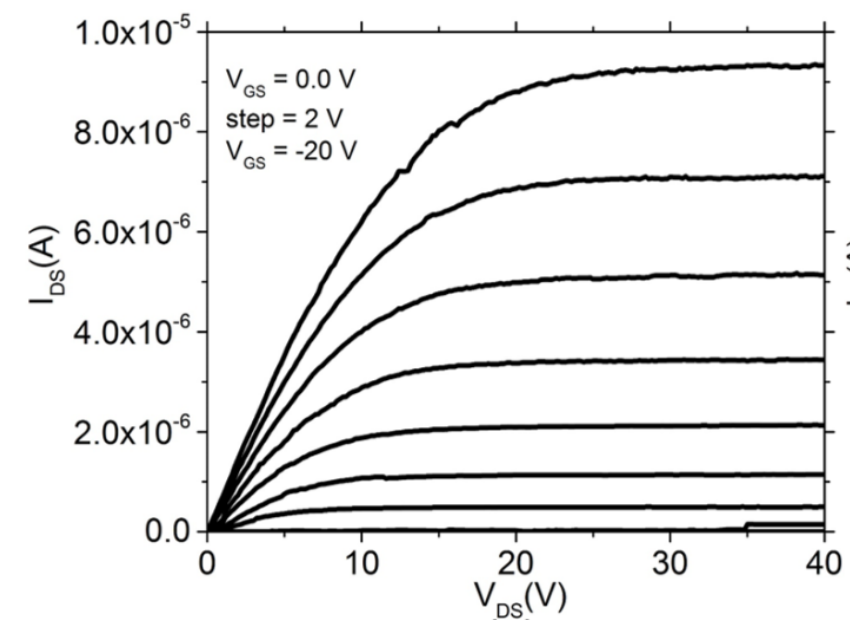

(a)

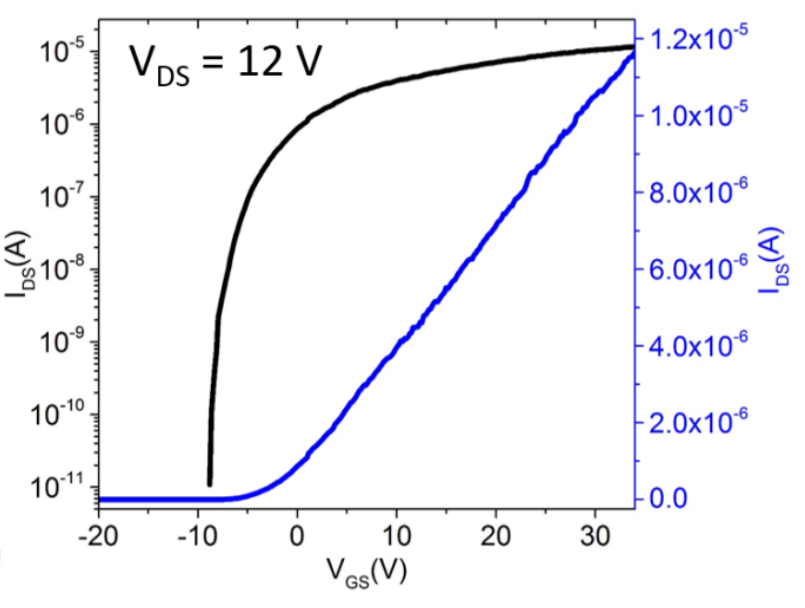

(b)

Fig. 3: Biosensor characteristics measured in air and darkness (a) $I_{D S} V_{D S}$ characteristics with a $V_{G S}$ drive from $-20 \mathrm{~V}$ to $0 \mathrm{~V}$ having steps of $2 \mathrm{~V}$ (b) $\mathrm{I}_{\mathrm{DS}} \mathrm{V}_{\mathrm{GS}}$ characteristics with $\mathrm{V}_{\mathrm{DS}}=12.0 \mathrm{~V}$. 
Fig. 4 shows the measured electrical characteristics of the biosensor in different bioanalyte solutions which were measured at $\mathrm{V}_{\mathrm{DS}}=12 \mathrm{~V}$ and top gate voltage (reference voltage) of $0 \mathrm{~V}$. Each environment within the experiment five measurements were made at time intervals of five minutes. The time interval between the different analyte solutions was one hour thirty minutes.

The five black curves (AirBefore) in Fig. 4 show the first set of electrical measurement in air. These five curves are clustered together and there is no overall trend concerning the voltage shift between the curves. Then five red curves (PBS) were measured as shown in Fig. 4 which are the second set of electrical measurement. These PBS curves are clustered closely together but tend to drift over time. They have a maximum voltage shift of $0.7 \mathrm{~V}$ at a fixed $\mathrm{I}_{\mathrm{DS}}$ of $20.0 \mathrm{nA}$. Fig. 4 (a) shows that PBS from AirBefore causes an average subthreshold shift in $\mathrm{V}_{\mathrm{GSsense}}$ from $-6.025 \mathrm{~V}$ to $+21.099 \mathrm{~V}$.

Then five blue curves (lysozyme from chicken white (LYSO: Fluka 62970)) were measured as shown in Fig. 4 which are the third set of electrical measurement. These lysozyme curves are clustered together but tend to drift over time. They have a maximum voltage shift of $0.88 \mathrm{~V}$ at a fixed IDs of 20.0 nA. Fig. 4 (a) shows that lysozyme from chicken white (LYSO: Fluka 62970) from PBS causes an average subthreshold shift in $\mathrm{V}_{\mathrm{GSsense}}$ from $21.099 \mathrm{~V}$ to $17.180 \mathrm{~V}$. The five brown curves (BSA) in Fig. 4 show the fourth set of electrical measurement. These bovine serum albumin (BSA: Sigma A3059) curves are clustered together but tend to drift over time. They have a maximum voltage shift of $0.7 \mathrm{~V}$ at a fixed $\mathrm{I}_{\mathrm{DS}}$ of $20.0 \mathrm{nA}$. Fig. 4 (a) shows that bovine serum albumin (BSA: Sigma A3059) from lysozyme from chicken white (LYSO: Fluka 62970) causes an average subthreshold shift in $\mathrm{V}_{\mathrm{GSsense}}$ from $17.180 \mathrm{~V}$ to $23.796 \mathrm{~V}$.

Finally the five green curves (AirAfter) in Fig. 4 show the fifth set of electrical measurement. These AirAfter curves are clustered together but tend to drift over time. They have a maximum voltage shift of $0.18 \mathrm{~V}$ at a fixed $\mathrm{I}_{\mathrm{DS}}$ of $20.0 \mathrm{nA}$. Fig. 4 (a) shows that AirAfter from bovine serum albumin (BSA: Sigma A3059) causes an average subthreshold shift in $\mathrm{V}_{\mathrm{GSsense}}$ from $23.796 \mathrm{~V}$ to $1.080 \mathrm{~V}$.

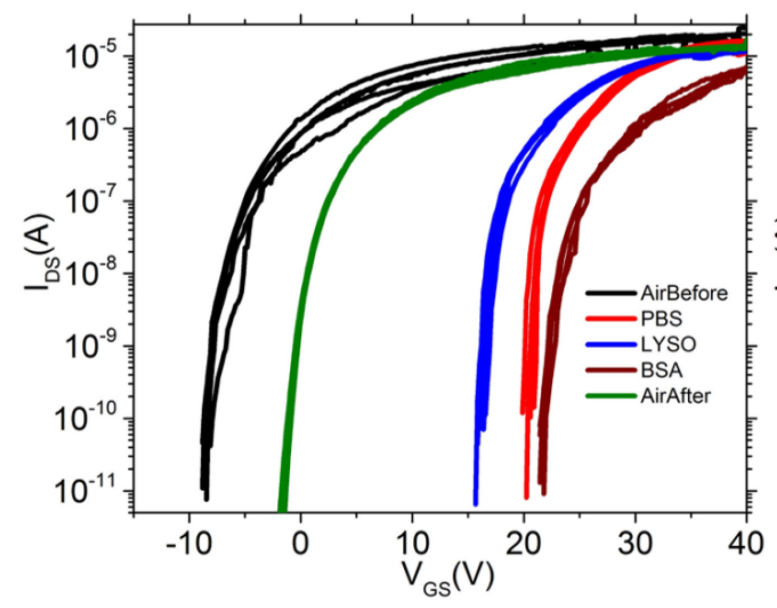

(a)

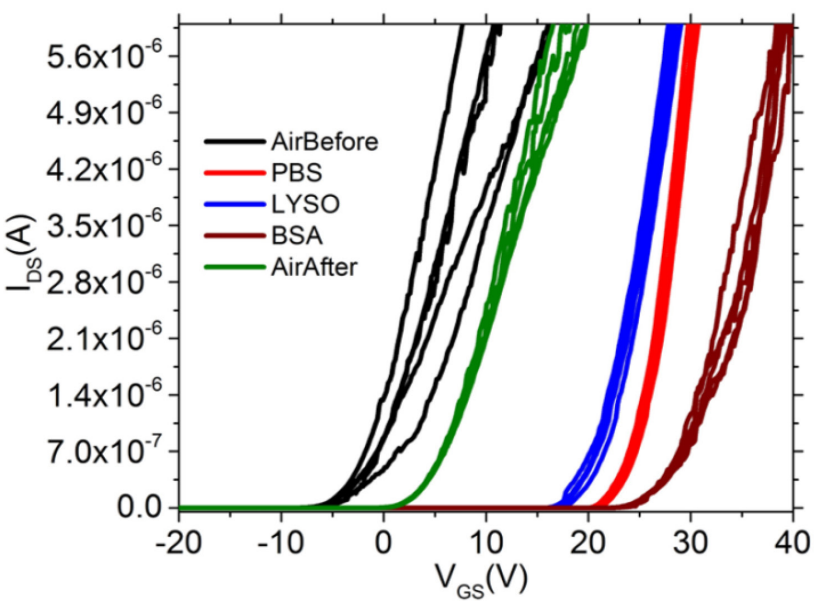

(b)

Fig. 4: Biosensor characteristics measured in air and darkness for different environments. These environments are AirBefore, PBS, LYSO, BSA, AirAfter (a) $\mathrm{I}_{\mathrm{DS}} \mathrm{V}_{\mathrm{GS}}$ subthreshold characteristics of the device (b) $\mathrm{I}_{\mathrm{DS}} \mathrm{V}_{\mathrm{GS}}$ linear characteristics of the device. All measurements are carried out at $\mathrm{V}_{\mathrm{DS}}=12.0 \mathrm{~V}$.

At fixed $\mathrm{I}_{\mathrm{DS}}=20.0 \mathrm{nA}$ and with reference to the PBS curve, the maximum net subthreshold voltage shifts due to protein binding for lysozyme from chicken white (LYSO: Fluka 62970) and bovine serum albumin (BSA: Sigma A3059) are $-4.4 \mathrm{~V}$ and $3.3 \mathrm{~V}$ respectively. The negative voltage shift of the LYSO indicates the presence of a positively charged protein and the positive voltage shift of the bovine serum albumin (BSA: Sigma A3059) signifies the presence of negatively charged protein. The $\mathrm{ZnO}$ NW biosensor has therefore distinguished between the positively charged lysozyme from chicken white (LYSO: Fluka 62970) and the negatively charged bovine serum albumin (BSA: Sigma A3059). 


\section{Discussion}

Table 2 discusses the low doped device. The average sensitivity for sensing of lysozyme from chicken white (LYSO: Fluka 62970) protein is $417.8 \%$ and the average sensitivity for the sensing of bovine serum albumin (BSA: Sigma A3059) protein is 5,765.8 \%. These values are high when compared with literature [1-27], which means that the device is highly sensitive. The low doping concentration of $2.0 \times 10^{16} \mathrm{~cm}^{-3}$ used for the NWFET channel is attributed to the high sensitivity results $[1,20-22]$. This trend can be qualitatively explained by equation 1 , which shows that the sensitivity is inversely proportional to the doping concentration [20].

Table 2: Summary of measurement of device sensitivity looking at voltage shift and current change between different solutions on the biosensor (low doped device).

\begin{tabular}{|l|l|l|l}
\hline \multicolumn{1}{|l}{ Low-doped Device Sensitivity } & $\Delta \mathrm{V}_{\mathrm{GSsense}}(\mathrm{V})$ & $\Delta \mathrm{I}(\mathrm{A})$ & $\begin{array}{l}\text { \% Change in } \\
\text { Sensitivity }\end{array}$ \\
\hline LYSO1 - PBS1 & $-3.849 \mathrm{~V}$ & $-1.1705 \times 10^{-6} \mathrm{~A}$ & $-293.2 \%$ \\
\hline LYSO2 - PBS2 & $-4.024 \mathrm{~V}$ & $-1.3721 \times 10^{-6} \mathrm{~A}$ & $-474.5 \%$ \\
\hline LYSO3 - PBS3 & $-3.674 \mathrm{~V}$ & $-1.1331 \times 10^{-6} \mathrm{~A}$ & $-457.0 \%$ \\
\hline LYSO4 - PBS4 & $-3.676 \mathrm{~V}$ & $-1.2836 \times 10^{-6} \mathrm{~A}$ & $-317.7 \%$ \\
\hline LYSO5 - PBS5 & $-4.375 \mathrm{~V}$ & $-1.5294 \times 10^{-6} \mathrm{~A}$ & $-543.2 \%$ \\
\hline BSA1 - PBS1 & $2.802 \mathrm{~V}$ & $3.9621 \times 10^{-7} \mathrm{~A}$ & $13,069 \%$ \\
\hline BSA2 - PBS2 & $2.276 \mathrm{~V}$ & $2.8133 \times 10^{-7} \mathrm{~A}$ & $3,582 \%$ \\
\hline BSA3 - PBS3 & $3.326 \mathrm{~V}$ & $2.4296 \times 10^{-7} \mathrm{~A}$ & $4,859 \%$ \\
\hline BSA4 - PBS4 & $2.800 \mathrm{~V}$ & $3.9447 \times 10^{-7} \mathrm{~A}$ & $4,106 \%$ \\
\hline BSA5 - PBS5 & $2.276 \mathrm{~V}$ & $2.7308 \times 10^{-7} \mathrm{~A}$ & $3,213 \%$ \\
\hline
\end{tabular}

The previous fabricated device [5] achieved maximum net subthreshold voltage shift of $340 \mathrm{mV}$ and $700 \mathrm{mV}$ for the LYSO and BSA solutions respectively. A NWFET sensitivity of $72 \%$ was achieved for the LYSO proteins while the BSA proteins resulted had a sensitivity of $98 \%$. These values are insignificant compared with those currently achieved. Comparing the low-doped device with the state-of-the-art devices shows that the device is more sensitive. Silicon is still the most used material for nanowire biosensors. The device made by X. Duan, et al., [29] shows the highest and stable sensitivity recorded in terms of 'voltage shift' having a value of $320 \mathrm{mV}$. A. Fulati, et al., [30] recorded a much lower value of voltage shift of $65.2 \mathrm{mV}$. The range of voltage sensitivity recorded by most researchers [1-43] for biosensors is between 50 and $400 \mathrm{mV}$. This paper records greater voltage shift $\left(\mathrm{V}_{\mathrm{GSsense}}\right)$ which makes the device more sensitive. The low doped device is more sensitive than X. Duan, et al., [29] by a value of $4.06 \mathrm{~V}$. The sensitivity is even much higher when compared with A. Fulati, et al., [30].

It must be noted that the charge-based measurements carried out are non-specific. The aim is to analysis the charge effect but in future the device will be functionalised to sense particular substances such as ions, proteins, DNA, and/or viruses. Antibody-functionalized nanowire sensors can be used to detect the proteins such as interleukin-8 (IL-8) and tumor necrosis factor-alpha (TNF- $\alpha$ ). Antibody-antigen dissociation constants must be in good agreement with low-salt enzyme-linked immunosorbent assays (ELISAs). 


\section{Conclusion}

A highly sensitive low-doped ZnO NWFET biosensor has been fabricated and measured. The low doped device had maximum sensitivity of $-543.2 \%$ for the PBS-LYSO protein and 13,069 \% for the PBS-BSA protein. The device response is stable and reproducible. The high sensitivity of the ZnO NWFET biosensor can be exploited for selective analyte detection by functionalizing the nanowire surface with antibodies or other biomolecular probe molecules.

\section{Acknowledgement}

N.M.J. Ditshego would like to acknowledge the Botswana International University of Science and Technology (BIUST) for supporting his doctoral studies and the Southampton Nanofabrication Centre for the experimental work. I would also like to acknowledge the EPSRC EP/K502327/1 grant support.

\section{Reference}

[1] M. Curreli, R. Zhang, F. N. Ishikawa, H. K. Chang, R. J. Cote, C. Zhou, and M. E. Thompson, "Real-time, label-free detection of biological entities using nanowire-based FETs," IEEE Trans. Nanotechnol., vol. 7, no. 6, Nov. 2008, pp. 651-667.

[2] A. Wei, L. Pan, and W. Huang, "Recent progress in the $\mathrm{ZnO}$ nanostructure-based sensors," Materials Science and Engineering B: Solid-State Materials for Advanced Technology, vol. 176, no. 18, 2011, pp. 1409-1421.

[3] S. M. Sultan, N. J. Ditshego, R. Gunn, P. Ashburn, and H. M. Chong, "Effect of atomic layer deposition temperature on the performance of top-down $\mathrm{ZnO}$ nanowire transistors." Nanoscale Research Letters, vol. 9, no. 1, Jan. 2014, p. 517.

[4] N. M. J. Ditshego, K. Sun, I. Zeimpekis, P. Ashburn, M. R. R. de Planque, and H. M. H. Chong, "Effects of surface passivation on top-down $\mathrm{ZnO}$ nanowire transistors," Microelectron. Eng., vol. 145, Sep. 2015, pp. 91-95.

[5] N. M. J. Ditshego, N. A. B. Ghazali, M. Ebert, K. Sun, I. Zeimpekis, P. Ashburn, M. R. R. de Planque, and H. M. H. Chong, "ZnO nanowire-FET for charge-based sensing of protein biomolecules," in 2015 IEEE 15th International Conference on Nanotechnology (IEEE-NANO), 2015, pp. 801-804.

[6] A. Menzel, K. Subannajui, F. Güder, D. Moser, O. Paul, and M. Zacharias, "Multifunctional ZnO-Nanowire-Based Sensor,” Adv. Funct. Mater., vol. 21, no. 22, Nov. 2011, pp. 4342-4348.

[7] G. A. C. Jones, G. Xiong, and D. Anderson, "Fabrication of nanoscale ZnO field effect transistors using the functional precursor zinc neodecanoate directly as a negative electron beam lithography resist," J. Vac. Sci. Technol. B Microelectron. Nanom. Struct., vol. 27, no. 6, Nov. 2009 , p. 3164.

[8] J. H. Jun, B. Park, K. Cho, and S. Kim, "Flexible TFTs based on solution-processed ZnO nanoparticles.," Nanotechnology, vol. 20, no. 50, Dec. 2009, p. 505201.

[9] M. Lee, K. Y. Baik, M. Noah, Y.-K. Kwon, J.-O. Lee, and S. Hong, "Nanowire and nanotube transistors for lab-on-a-chip applications.," Lab Chip, vol. 9, no. 16, Aug. 2009, pp.2267-80.

[10] C. Yang, C. $\mathrm{Xu}$, and $\mathrm{X}$. Wang, " $\mathrm{ZnO} / \mathrm{Cu}$ nanocomposite: a platform for direct electrochemistry of enzymes and biosensing applications.," Langmuir, vol. 28, no. 9, Mar. 2012, pp. $4580-5$.

[11] K. Sun, I. Zeimpekis, M. Lombardini, N. M. J. Ditshego, S. J. Pearce, K. S. Kiang, O. Thomas, M. R. R. De Planque, H. M. H. Chong, H. Morgan, and P. Ashburn, "Three-mask polysilicon thin-film transistor biosensor," IEEE Trans. Electron Devices, 2014, pp. 2170 - 2176. 
[12] N. A. B. Ghazali, M. Ebert, N. M. J. Ditshego, M. R. R. de Planque, and H. M. H. Chong, "Top-down fabrication optimisation of $\mathrm{ZnO}$ nanowire-FET by sidewall smoothing," Microelectron. Eng., vol. 159, Jun. 2016, pp. 121-126.

[13] K. Sun, I. Zeimpekis, C. Hu, N. M. J. Ditshego, O. Thomas, M. R. R. De Planque, H. M. H. Chong, H. Morgan, and P. Ashburn, "Low-cost top-down zinc oxide nanowire sensors through a highly transferable ion beam etching for healthcare applications," Microelectronic Engineering, vol. 153, 2015, pp. 96-100.

[14] P.-Y. Yang, J.-L. Wang, P.-C. Chiu, J.-C. Chou, C.-W. Chen, H.-H. Li, and H.-C. Cheng, " $\mathrm{pH}$ Sensing Characteristics of Extended-Gate Field-Effect Transistor Based on Al-Doped ZnO Nanostructures Hydrothermally Synthesized at Low Temperatures," IEEE Electron Device Lett., vol. 32, no. 11, Nov. 2011, pp. 1603-1605.

[15] N. Batra, M. Tomar, and V. Gupta, "Realization of an efficient cholesterol biosensor using ZnO nanostructured thin film.," Analyst, vol. 137, no. 24, Dec. 2012, pp. 5854-9.

[16] Y.-C. Shen, C.-H. Yang, S.-W. Chen, S.-H. Wu, T.-L. Yang, and J.-J. Huang, "IGZO thin film transistor biosensors functionalized with $\mathrm{ZnO}$ nanorods and antibodies.," Biosens. Bioelectron., vol. 54, Apr. 2014, pp. 306-10.

[17] A. Wei, X. W. Sun, J. X. Wang, Y. Lei, X. P. Cai, C. M. Li, Z. L. Dong, and W. Huang, "Enzymatic glucose biosensor based on $\mathrm{ZnO}$ nanorod array grown by hydrothermal decomposition,” Appl. Phys. Lett., vol. 89, no. 12, Sep. 2006, p. 123902.

[18] R. Ahmad, N. Tripathy, and Y.-B. Hahn, "Wide linear-range detecting high sensitivity cholesterol biosensors based on aspect-ratio controlled $\mathrm{ZnO}$ nanorods grown on silver electrodes," Sensors Actuators B Chem., vol. 169, Jul. 2012, pp. 382-386.

[19] M. Ahmad, C. Pan, Z. Luo, and J. Zhu, "A single ZnO nanofiber-based highly sensitive amperometric glucose biosensor,” J. Phys. Chem. C, vol. 114, no. 20, May 2010, pp. 9308-9313.

[20] B. Reddy, B. R. Dorvel, J. Go, P. R. Nair, O. H. Elibol, G. M. Credo, J. S. Daniels, E. K. C. Chow, X. Su, M. Varma, M. a. Alam, and R. Bashir, "High-k dielectric Al2O3 nanowire and nanoplate field effect sensors for improved $\mathrm{pH}$ sensing," Biomed. Microdevices, vol. 13, no. 2, Apr. 2011, pp. 335-344.

[21] K. Chen, B. Li, and Y. Chen, "Silicon nanowire field-effect transistor-based biosensors for biomedical diagnosis and cellular recording investigation," Nano Today, vol. 6, no. 2, Apr. 2011, pp. 131-154.

[22] M.-Y. Shen, B.-R. Li, and Y.-K. Li, "Silicon nanowire field-effect-transistor based biosensors: from sensitive to ultra-sensitive.," Biosens. Bioelectron., vol. 60, Oct. 2014, pp. 101-11.

[23] N. Zhang, K. Yu, Q. Li, Z. Q. Zhu, and Q. Wan, "Room-temperature high-sensitivity H[sub 2]S gas sensor based on dendritic $\mathrm{ZnO}$ nanostructures with macroscale in appearance," J. Appl. Phys., vol. 103, no. 10, May 2008, p. 104305.

[24] S. K. Yoo, S. K. Yoo, I. Hwang, I. Hwang, B. P. Mun, B. P. Mun, J. H. Lee, and J. H. Lee, "High sensitivity ph sensing using schottky contacted silicon nanowire field-effect transistor 1," October, vol. 1, no. d, 2008, pp. 323-325.

[25] J. Zhou, N. S. Xu, and Z. L. Wang, "Dissolving Behavior and Stability of ZnO Wires in Biofluids: A Study on Biodegradability and Biocompatibility of $\mathrm{ZnO}$ Nanostructures," Adv. Mater., vol. 18, no. 18, Sep. 2006, pp. 2432-2435.

[26] S. Chen, J. G. Bomer, W. G. van der Wiel, E. T. Carlen, and A. van den Berg, "Top-down fabrication of sub-30 nm monocrystalline silicon nanowires using conventional microfabrication.," ACS Nano, vol. 3, no. 11, Nov. 2009, pp. 3485-92. 
[27] C. G. Kang, J. W. Kang, S. K. Lee, S. Y. Lee, C. H. Cho, H. J. Hwang, Y. G. Lee, J. Heo, H.-J. Chung, H. Yang, S. Seo, S.-J. Park, K. Y. Ko, J. Ahn, and B. H. Lee, "Characteristics of CVD graphene nanoribbon formed by a ZnO nanowire hardmask.," Nanotechnology, vol. 22, no. 29, Jul. 2011, p. 295201.

[28] R. Chen, W. Zhou, and M. Zhang, "Self-Aligned Indium-Gallium-Zinc Oxide Thin-Film Transistor With Phosphorus-Doped Source/Drain Regions," ... Device Lett. IEEE, vol. 33, no. 8, Aug. 2012, pp. 1150-11521152.

[29] X. Duan, Y. Li, N. K. Rajan, D. a Routenberg, Y. Modis, and M. a Reed, "Quantification of the affinities and kinetics of protein interactions using silicon nanowire biosensors.," Nat. Nanotechnol., vol. 7, no. 6, Jun. 2012, pp. 401-7.

[30] A. Fulati, S. M. U. Ali, M. H. Asif, N. ul H. Alvi, M. Willander, C. Brännmark, P. Strålfors, S. I. Börjesson, F. Elinder, and B. Danielsson, "An intracellular glucose biosensor based on nanoflake ZnO,” Sensors Actuators B Chem., vol. 150, no. 2, Oct. 2010, pp. 673-680. 